\title{
ON THE ASSESSMENT OF SIMILARITY OF DRUG DISSOLUTION PROFILES- A SIMULATION STUDY
}

\author{
HUEY LIN JU \\ Associate Professor and Director, Statistical Consulting Laboratory, Department of Statistics, \\ National Chengchi University, Taipei, Taiwan \\ SHU-JEAN LIAW \\ Research Assistant, Department of Psychiatry, National Taiwan University Hospital, Taipei, Taiwan
}

\begin{abstract}
In vitro dissolution has been recognized as an important element in drug development to assure the quality of drug products. It determines the rate and extent of the drug release and could be used as a surrogate for bioequivalence (BE) trials under certain conditions. Although methods for assessing the similarity of dissolution profiles between two drug products are available in the literature, most of them do not provide strong statistical and/or scientific justifications. This paper examines the properties of some commonly used methods and compares the results via a simulation study under the situations where the dissolution profile can be approximated by a linear or quadratic relationship over time. The similarity factor stated in the United States Food and Drug Administration (FDA) guideline (1) and the time series approach proposed by Chow (2) are also included. The results show that Chow's method is very sensitive to the variation in the amount dissolved. Both methods are more relaxed and have a lower probability of declaring dissimilarity than the others.
\end{abstract}

Key Words: Dissolution; Bioequivalence; ANOVA; Split-plot; Time series

\section{INTRODUCTION}

IN VITRO DISSOLUTION HAS been recognized as an important element in drug development. It can not only determine the rate and extent of the drug release, but also assure that the drug product can meet the United States Pharmacopoeia and National Formulary (USP/NF) standards for identity, strength, quality, and purity (3). In addition, it can be used as a surrogate for assessment of bioequivalence under certain conditions.

For dissolution testing, as stated in the USP/NF, the rotating basket and paddle are two of the most commonly adapted apparatus for the testing. In USP III, a three-stage sampling plan is specified to determine whether the test results meet the acceptance criteria (4). In the recent scale-up and postapproval changes (SUPAC) guideline issued by the FDA, the dissolution testing is classified into cases A, B, and C (1). Case A is the dissolution of $Q$ $=85 \%$ in 15 minutes in $900(\mathrm{~mL})$ of $0.1 \mathrm{HCl}$, using a rotating basket at $100 \mathrm{rpm}$ or a paddle

Reprint address: Huey Lin Ju, Associate Professor and Director, Statistical Consulting Laboratory, Department of Statistics, National Chengchi University, 64, Sec 2, Chi-Nan Rd., Taipei, Taiwan, 11623. 
at $50 \mathrm{rpm}$. Case B is the multipoint dissolution profiles in the application/compendia medium at $15,30,45,60$, and 120 minutes or until an asymptote is reached for the proposed and currently accepted formulation. Case $\mathrm{C}$ is the multipoint dissolution profiles performed in water, $0.1 \mathrm{~N} \mathrm{HCl}$, and USP buffer media at $\mathrm{pH}=4.5,6.5$, and 7.5 for five separate profiles for the proposed and currently accepted formulations. Sampling time is specified as Case B or until either $90 \%$ of the drug from the drug product is dissolved or an asymptote is reached. The requirement for the dissolution testing for different level changes in components and composition, site, batch size, and manufacturing (including equipment and process) is summarized in Table 1.

To compare dissolution profiles between two drug products, several methods have been proposed. These methods, by the nature of the procedures, could be classified as model dependent (curve fitting) and model independent (statistical analysis). The commonly used models in the curve fitting procedures include exponential (5), probit, Gompertz (6), logistic (7), Weibull (8), and the three control factor models (9). For the curve fitting procedure, it is assumed that the dissolution profiles can be expressed by the selected curve. The procedure is first to fit the curve of the response of the experiment (eg, Time to d percentage dissolved $(\mathrm{T} \% \mathrm{~d})$, percentage dissolved to time $\mathrm{t}(\% \mathrm{Dt})$, and area under the curve (AUCdiss.), and the relative efficiency) and compute the associated parameters of the model for both reference and test, respectively. It is then followed by comparing the corresponding parameters from the two curves. For the curve fitting procedure, how to select an appropriate curve is critical. If the curve selected is inappropriate, it will be misleading to compare the dissolution profiles by testing the parameters from the two models. In other words, the error of making an incorrect conclusion could actually occur in two ways: selecting the wrong model or making the wrong judgment when comparing the corresponding parameters. Therefore, the lack-offit test is essential before comparing the associated parameters.

The most commonly used statistical methods include moment based comparison, individual time point test, two-way ANOVA, ANCOVA (10), analysis of first difference (11), repeated measurement split-plot (12), multivariate analysis (13), and so forth. These methods do not require the preset curve of the profiles. Some analyses, however, have an underlying assumption that requires the responses to be independent. This assumption could not be applied to dissolution testing since the amount dissolved over time within the same drug product is in fact correlated. Although the multivariate analysis takes into account the

TABLE 1

SUPAC-IR Dissolution Testing Requirements

\begin{tabular}{|c|c|c|c|}
\hline \multirow[b]{2}{*}{ Change } & \multicolumn{3}{|c|}{ Levels } \\
\hline & 1 & 2 & 3 \\
\hline $\begin{array}{l}\text { Components \& } \\
\text { Composition }\end{array}$ & $\begin{array}{l}\text { Application/Compendial } \\
\text { Requirement }\end{array}$ & $\begin{array}{l}\text { 1) High permeability, High Solubility: } \\
\text { A (fails } \rightarrow \text { B or C) } \\
\text { 2) Low permeability, High Solubility : B } \\
\text { 3) High permeability, Low Solubility : C }\end{array}$ & $\mathrm{B}$ \\
\hline Site & $\begin{array}{l}\text { Application/Compendial } \\
\text { Requirement }\end{array}$ & Application/Compendial Requirement & $\mathrm{B}$ \\
\hline Batch size & $\begin{array}{l}\text { Application/Compendial } \\
\text { Requirement }\end{array}$ & $\mathrm{B}$ & \\
\hline $\begin{array}{l}\text { Manufacturing } \\
\text {-Equipment }\end{array}$ & $\begin{array}{l}\text { Application/Compendial } \\
\text { Requirement }\end{array}$ & $\mathrm{C}$ & \\
\hline -Process & $\begin{array}{l}\text { Application/Compendial } \\
\text { Requirement }\end{array}$ & B & $\mathrm{B}$ \\
\hline
\end{tabular}


correlation problem, the procedure is fairly complicated. Moreover, if the time point selected is large (eg, larger than 3), it is difficult to interpret the results. In the SUPAC guideline (1), FDA indicates that dissolution profiles could be compared based on a similarity factor. Although this method is easy to apply, it lacks scientific justification. Recently, Chow (2) used the concept of bioequivalence and developed a procedure by utilizing a time series approach (14). This method takes into account the correlation structure of the amount dissolved over time and evaluates the equivalence in the dissolution profiles.

The objective of this paper is to evaluate the properties and investigate the implications of the most commonly used methods (eg, ANCOVA, two-way ANOVA, split-plot) in comparing dissolution profiles although the former two methods should not be used in theory. The performance of these methods is compared with the similarity factor of FDA and Chow's methods via a simulation study under the assumption that the dissolution profile can be approximated by a linear or quadratic relationship over time. In the next section, methods of similarity factor, split-plot, and Chow will be briefly outlined, followed by the simulation results. A summary and discussion is presented in the last section.

\section{METHODS}

\section{The Similarity Factor of FDA}

In the FDA guideline, dissolution profiles may be compared by using the similarity factor $f_{2}$ as follows (1):

$$
f_{2}=50 \log _{10}\left\{100\left[1+\frac{1}{t} \sum_{j=1}^{t}\left(R_{j}-T_{j}\right)^{2}\right]^{-0.5}\right\}
$$

where $R_{j}$ and $T_{j}$ represent the average percent dissolved at time $\mathrm{j}$ for reference and test, respectively, and $\mathrm{t}$ is the number of time points tested. It can be seen that $f_{2}$ is in fact between the interval of $(-\infty, 100)$. If the average dissolution amount between $\mathrm{R}$ and $\mathrm{T}$ is identical at each time point (ie, $\Sigma_{j=1}^{t}\left(R_{j}-T_{j}\right)^{2}=0$ ), then $f_{2}=100$. FDA defines that when $f_{2}$ is between 50 (where $\Sigma_{j=1}^{t}\left(R_{j}-T_{j}\right)^{2}=99$ ) and 100, then similarity of the dissolution profiles between two drug products could be claimed.

\section{The Spilt-Plot Approach of Gill}

Dissolution profiles could also be compared by using Gill's split-plot trend analysis. The analysis starts with the model (12):

$$
\begin{aligned}
Y_{i j k}=\mu+\tau_{i}+N_{k(i)}+T_{j}+(\tau T)_{i j}+\varepsilon_{i j k} \quad i & =1,2 ; j=1,2, \ldots, t ; \\
k & =1,2, \ldots, n
\end{aligned}
$$

where $Y_{i j k}$ is the amount dissolved in the $k$ th location (basket) of treatment $i(i=1$ or 2$)$ at time $j, \mu$ is the overall mean, $\tau_{i}$ is the $i$ th treatment effect, $N_{k(i)}$ is the $k$ th location effect nested in treatment $i$, assuming a normal distribution with mean 0 and variance $\sigma^{2}, T_{j}$ is the $j$ th time effect, $(\tau T)_{i j}$ is the interaction between treatment $i$ and time $j$, and $\varepsilon_{i j k}$ is the random error term, assuming a normal distribution with mean 0 and $\operatorname{corr}\left(\varepsilon_{i j k}, \varepsilon_{i j}{ }^{1}{ }_{k}\right)=\rho$.

In equation (2), the interaction is first examined by the following hypotheses:

$$
\begin{array}{ll} 
& H_{0}:(\tau T)_{i j}=0, \forall i, j \\
\text { vs. } \quad H_{1}: \text { at least one }(\tau T)_{i j} \neq 0 .
\end{array}
$$


If $H_{0}$ is rejected, then the dissimilarity of dissolution profiles $\mathrm{T}$ and $\mathrm{R}$ is concluded. If $H_{0}$ is not rejected, equation (2) is reduced to:

$$
\begin{aligned}
& Y_{i j k}=\mu+\tau_{i}+N_{k(i)}+T_{j}+\varepsilon_{i j k} \quad i=1,2 ; j=1,2, \ldots, t ; \\
& k=1,2, \ldots, n \text {. }
\end{aligned}
$$

Thus, the main effect can be evaluated by testing:

$$
\begin{aligned}
& H_{0}: \tau_{1}=\tau_{2}=0 \\
\text { vs. } & H_{1}: \text { at least one } \tau_{i} \neq 0 .
\end{aligned}
$$

If $H_{0}$ is rejected, then the dissimilarity of dissolution profiles $\mathrm{T}$ and $\mathrm{R}$ is concluded, otherwise, similarity is achieved.

The above test procedures are also applied to ANCOVA and two-way ANOVA models.

\section{The Time Series Approach of Chow}

Chow uses the concept of bioequivalence to define the similarity limit for dissolution testing as $\left(\delta_{L}, \delta_{U}\right)$, where $\delta_{L}=\frac{Q-\delta}{Q+\delta}, \delta_{U}=\frac{Q+\delta}{Q-\delta}$, and $Q$ is the amount of dissolved active ingredient specified in the individual monograph in the USP/NF. Two drug products are claimed to have similar dissolution profiles if the ratio of dissolution rates is within $\left(\delta_{L}, \delta_{U}\right)$ with $95 \%$ assurance. Chow utilizes the auto correlation (1) time series model to assess the similarity as follows:

$$
R_{j k}=\gamma_{j k}+\phi_{k}\left(R_{(j-1) k}-\gamma_{(j-1) k}\right)+\varepsilon_{j k} j=2, \ldots, t ; k=1,2, \ldots, n
$$

where $R_{j k}$ is the ratio of dissolution rate between test and reference at basket k and time $\mathrm{j}$, $\gamma_{j k}$ is the true relative dissolution rate at basket $\mathrm{k}$ and time $j, \phi_{k}$ is the degree of correlation between consecutive measurements, and $\varepsilon_{i j k}$ is the random error deviates, assuming a normal distribution with mean 0 and variance $\sigma^{2}$.

In practice, since $\gamma_{j k}$ usually differs in time and location, it is assumed that $\gamma_{j k}$ is a normally distributed random variable with mean $\gamma$ and variance $\tau^{2}$. The $(1-\alpha) \times 100 \%$ confidence interval of $\gamma$, denoted by $(L, U)$, can be computed as:

$$
\begin{aligned}
& L=\hat{\gamma}-z_{(1-\alpha / 2)}(\sqrt{\operatorname{Var}(\hat{\gamma})}+\tau) \\
& U=\hat{\gamma}+z_{(1-\alpha / 2)}(\sqrt{\operatorname{Var}(\hat{\gamma})}+\tau)
\end{aligned}
$$

where

$$
\begin{gathered}
\hat{\gamma}=\frac{1}{n t} \sum_{k=1}^{n} \sum_{j=1}^{t} R_{j k} \\
\hat{\tau}^{2}=\frac{1}{n t-1} \sum_{k=1}^{n} \sum_{j=1}^{t}\left(R_{j k}-\bar{R}\right)^{2}-\frac{1}{n(t-1)} \sum_{k=1}^{n} \sum_{j=1}^{t}\left(R_{j k}-\bar{R}_{k}\right)^{2} \\
\mathrm{Z}_{(1-\alpha / 2)} \text { is the }(1-\alpha / 2) 100 \% \text { quantile of normal distribution } \\
\operatorname{Var}(\hat{\gamma})=\frac{1}{n^{2} t} \sum_{k=1}^{n} \hat{\psi}_{k}^{2}\left(1+2 \sum_{p=1}^{t-1} \frac{t-p}{t} \hat{\phi}_{k}^{p}\right)+\frac{\hat{\tau}^{2}}{n t}
\end{gathered}
$$


and

$$
\begin{aligned}
\hat{\Psi}_{k}^{2} & =\frac{1}{t-1} \sum_{j=1}^{t}\left(R_{j k}-\bar{R}_{k}\right)^{2} \\
\hat{\phi}_{k} & =\frac{\sum_{j}^{t-1}\left(R_{j k}-\bar{R}_{k}\right)\left(R_{(j+1) k}-\bar{R}_{k}\right)}{\sum_{j=1}^{t}\left(R_{j k}-\bar{R}_{k}\right)^{2}} \\
\bar{R}_{k} & =\frac{1}{t} \sum_{j=1}^{t} R_{j k} .
\end{aligned}
$$

If $(L, U)$ is within the interval of $\left(\delta_{L}, \delta_{U}\right)$, then similarity between the test and reference products is claimed.

The above procedure is used to determine global similarity. In the paper, Chow also derives a formula to examine the local similarity at each time point (2).

\section{An Example}

To illustrate the above methods, consider the example given in Chow (2) which was taken from Tsong and Hammerstrom (5).The test results are shown in Table 2.

It can be seen that for Chow's method, similarity is claimed if $Q \leq 70 \%$. Since $f_{2}$ has the value of 63.59 which is between 50 and 100 , the data are also claimed to be similar. Splitplot, ANCOVA, and two-way ANOVA, however, have significant interactions between treatment and time, therefore, similarity cannot be determined by using these three methods.

\section{A SIMULATION STUDY}

To evaluate the performance of the methods, a simulation study was conducted. Although most of the dissolution profiles are nonlinear, it could be approximated by the first and second order polynomials via Taylor's expansion, assuming that higher order terms are negligible. Therefore, linear and quadratic models within a given range are considered to evaluate the properties of these methods in the simulation study. In each of the two models, data were generated 1,000 times and the probability of concluding dissimilarity of the dissolution profiles was computed.

\begin{tabular}{|c|c|c|c|c|c|c|c|}
\hline \multicolumn{8}{|c|}{ Methods } \\
\hline \multicolumn{2}{|c|}{ Two-Way ANOVA } & \multicolumn{2}{|c|}{ ANCOVA } & \multicolumn{2}{|c|}{ Split-Plot } & FDA & Chow \\
\hline \multicolumn{2}{|c|}{$p$-value } & \multicolumn{2}{|c|}{$p$-value } & \multicolumn{2}{|c|}{$p$-value } & Similarity & Time Series \\
\hline interaction & $\begin{array}{c}\text { no } \\
\text { interaction } \\
\text { Trt }\end{array}$ & $\begin{array}{l}\text { unequal } \\
\text { slope }\end{array}$ & $\begin{array}{l}\text { equal } \\
\text { slope } \\
\text { Trt }\end{array}$ & interaction & $\begin{array}{c}\text { no } \\
\text { interaction } \\
\text { Trt }\end{array}$ & & $\mathrm{Cl}$ \\
\hline 0.0001 & & 0.0001 & & 0.0001 & & $63.59^{*}$ & $\begin{array}{c}(86.9,112.7)^{*} \\
Q \leq 70 \%\end{array}$ \\
\hline
\end{tabular}

TABLE 2

Results from Various Methods Using Data from Tsong and Hammestrom (1992)

*Similarity between two drug profiles is claimed. 


\section{Case 1: Linear}

Consider the case where the dissolution profiles for reference and test products can be described by the following linear models:

$$
\begin{aligned}
& R=a_{1}+b_{1} t+\varepsilon_{1} \\
& T=a_{2}+b_{2} t+\varepsilon_{2}
\end{aligned}
$$

where $\varepsilon_{1}$ and $\varepsilon_{2}$ are normally distributed random variables with mean 0 and variance $\sigma^{2}$. Two sets of time points are considered, namely:

1. $\mathrm{t}=0,0.2,0.4,0.6,0.7,0.8(\mathrm{hr})$, and

2. $\mathrm{t}=0.1,0.2,0.4,0.6,0.7,0.8(\mathrm{hr})$.

$a_{1}$ and $b_{1}$ are selected to be 0 and 100, respectively, so that the reference will dissolve $75 \%$ at 45 minutes. In this setting, how sensitive the probability is to the change of intercept, slope, and the variation is investigated.

1. Variance $\left(\sigma^{2}\right)$ varied $\left(1 \leq \sigma \leq 60, a_{1}=a_{2}=0, b_{1}=b_{2}=100\right)$-Figure 1.a shows that unless the variation is very large, the probability of declaring dissimilarity for the similarity

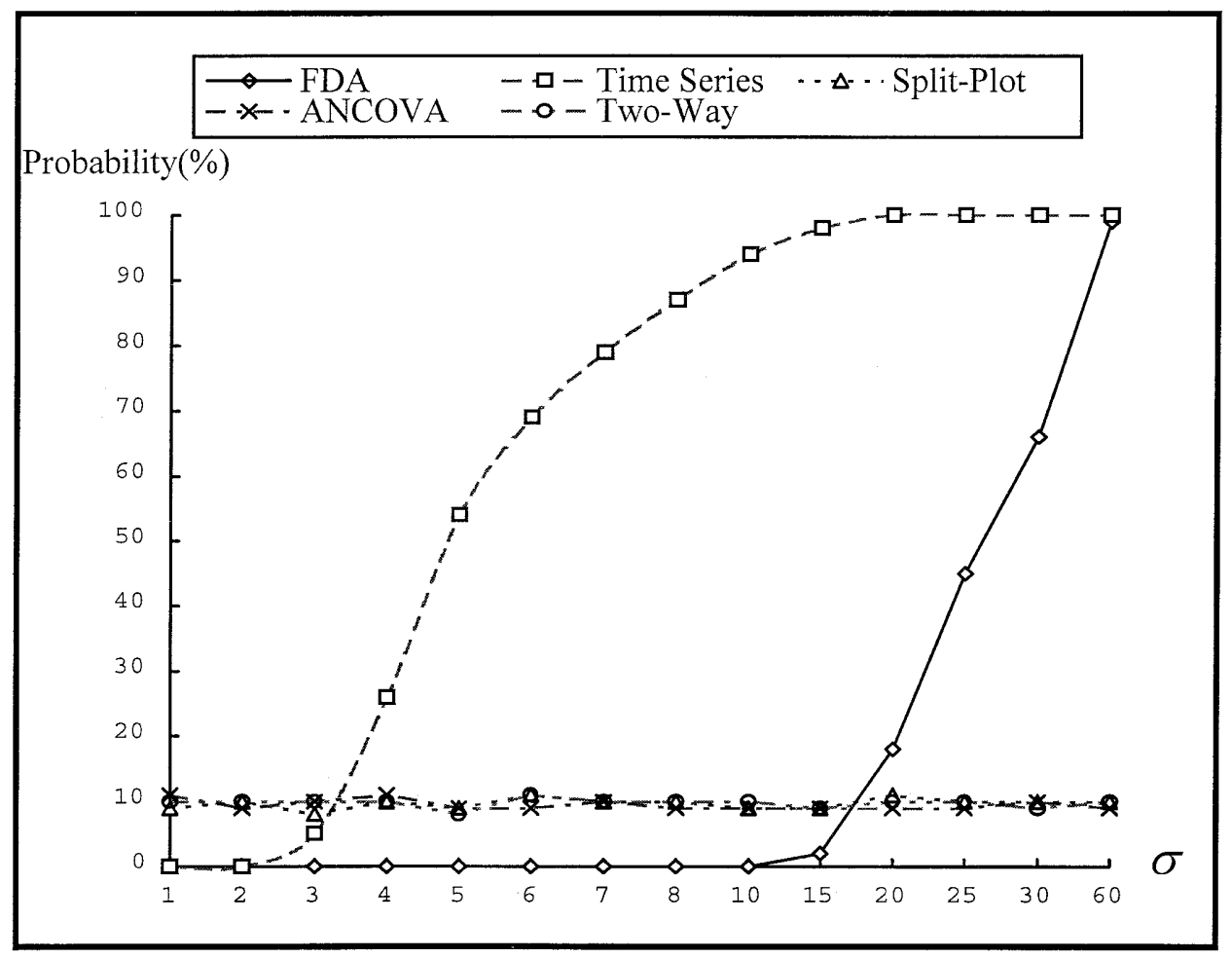

FIGURE 1a. The probability of concluding dissimilarity. $a_{1}=\sigma_{2}=0, b_{1}=b_{2}=100,1 \leq \sigma \leq$ $60,0.1 \leq r \leq 0.8$. 
factor is zero. The probability of Chow's method is an increasing function of $\sigma$ while it is a constant $(10 \%)$ for split-plot, ANCOVA, or two-way ANOVA regardless of the variation changes. The later corresponds to the Type I error used in the simulation since the dissimilarity could occur either when the interaction exists or when the treatment effects differ (without an interaction model), each with a 5\% significance level. The probability of declaring dissimilarity between two drug products for these methods could be observed as:

$$
\begin{array}{cl}
\quad \sigma \leq 3 & \text { FDA }<\text { Chow }<\text { others } \\
3<\sigma \leq 15 & \text { FDA }<\text { others }<\text { Chow } \\
15<\sigma & \text { other }<\text { FDA }<\text { Chow } .
\end{array}
$$

Figure 1.b shows that when time 0 is included, Chow's method will declare dissimilarity for almost $100 \%$ regardless of the variation. This phenomenon is observed throughout the simulations. It can be explained that Chow's test statistic is, in fact, a ratio of two random deviates. If the denominator is close to 0 , then $\mathrm{R}$ is large. Therefore, the probability of concluding dissimilarity is large,

2. Intercept $\left(a_{2}\right)$ varies $\left(-10 \leq a_{2} \leq 10, b_{1}=b 2=100\right)$-This occurs in the case where there are differences between $\mathrm{T}$ and $\mathrm{R}$ in the initial dissolving ( 0.1 hour) but at the same rate neither the Chow nor the FDA method are sensitive to the change while other methods declare dissimilarity with $100 \%$ even when the change is only $2.5 \%$ apart (Figure 2). The probability of concluding dissimilarity could be observed as:

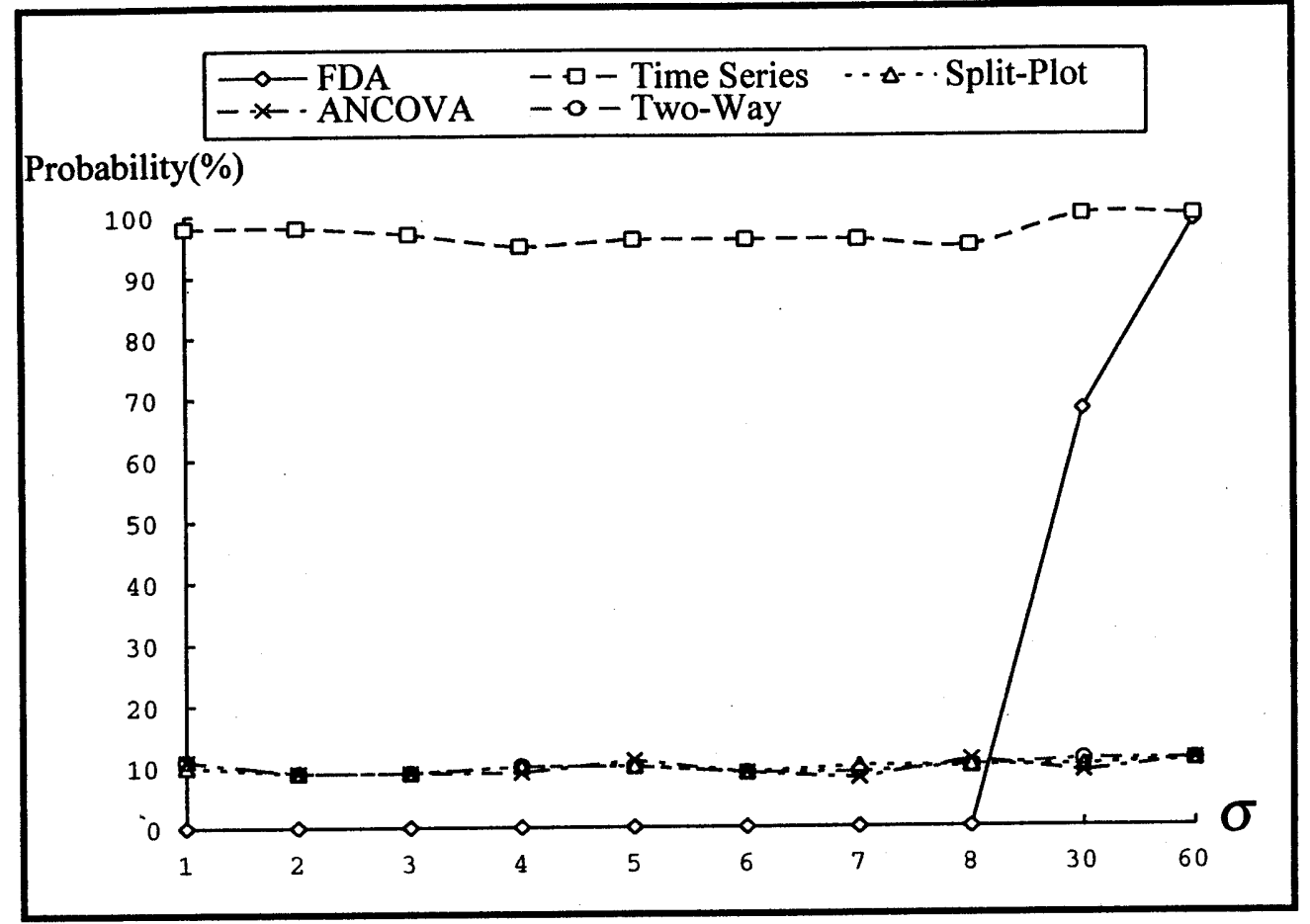

FIGURE 1b. The probability of concluding dissimilarity. $a_{1}=a_{2}=0, b_{1}=b_{2}=100,1 \leq \sigma \leq$ $60,0 \leq t, 0.8$. 


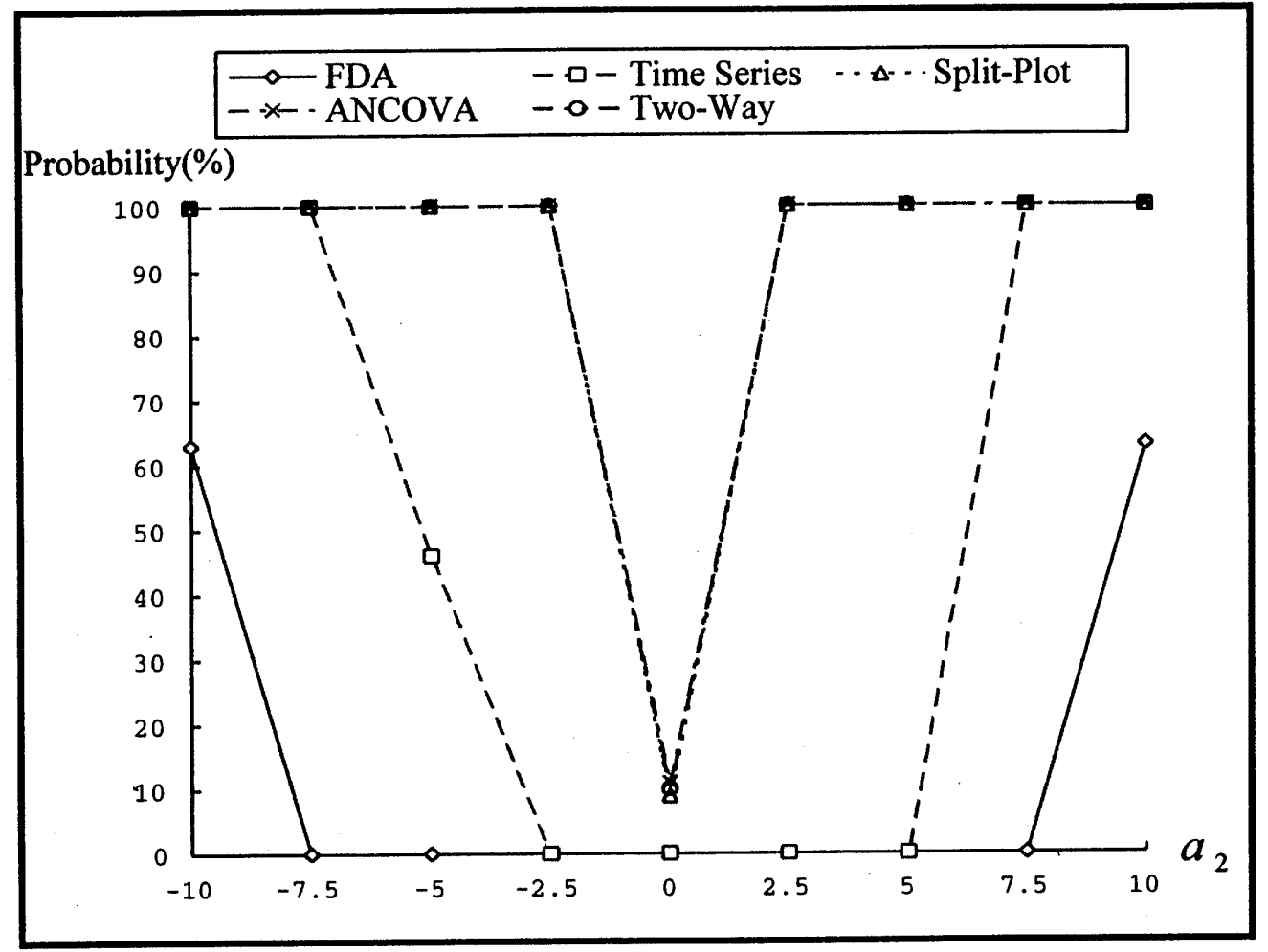

FIGURE 2. The probability of concluding dissimilarity. $a_{1}=0, b_{1}=b_{2}=100, \sigma=1,0.1 \leq$ $t \leq 0.8$.

FDA $<$ Chow $<$ others,

and

3. Slope $\left(b_{2}\right)$ varies $\left(80 \leq b_{2} \leq 120, a_{1}=a_{2}=0\right)$ - This is the same as the change in the intercept. Split-plot, ANCOVA, and two-way ANOVA observe the change right away and the probability jumps from about $10 \%$ (identical) to $100 \%$ even when the slope is only four units apart which results in $Q_{0.75}(T)=72.5 \%$ (Figure 3). Both the Chow and FDA methods are very insensitive to the change. For example, even when the test is $10 \%$ different from the reference at time $t=0.75$, both methods have zero probability to detect the dissimilarity, especially Chow's method. The probability is:

Chow $<$ FDA $<$ others.

\section{Case 2. Quadratic Model}

Consider the model

$$
\begin{aligned}
& R=a_{1}+b_{11} t+b_{12} t^{2}+\varepsilon_{1} \\
& T=a_{2}+b_{21} t+b_{22} t^{2}+\varepsilon_{2}
\end{aligned}
$$

where $\varepsilon_{1}$ and $\varepsilon_{2}$ have the same assumption as equations (3.1) and (3.2). The time points 


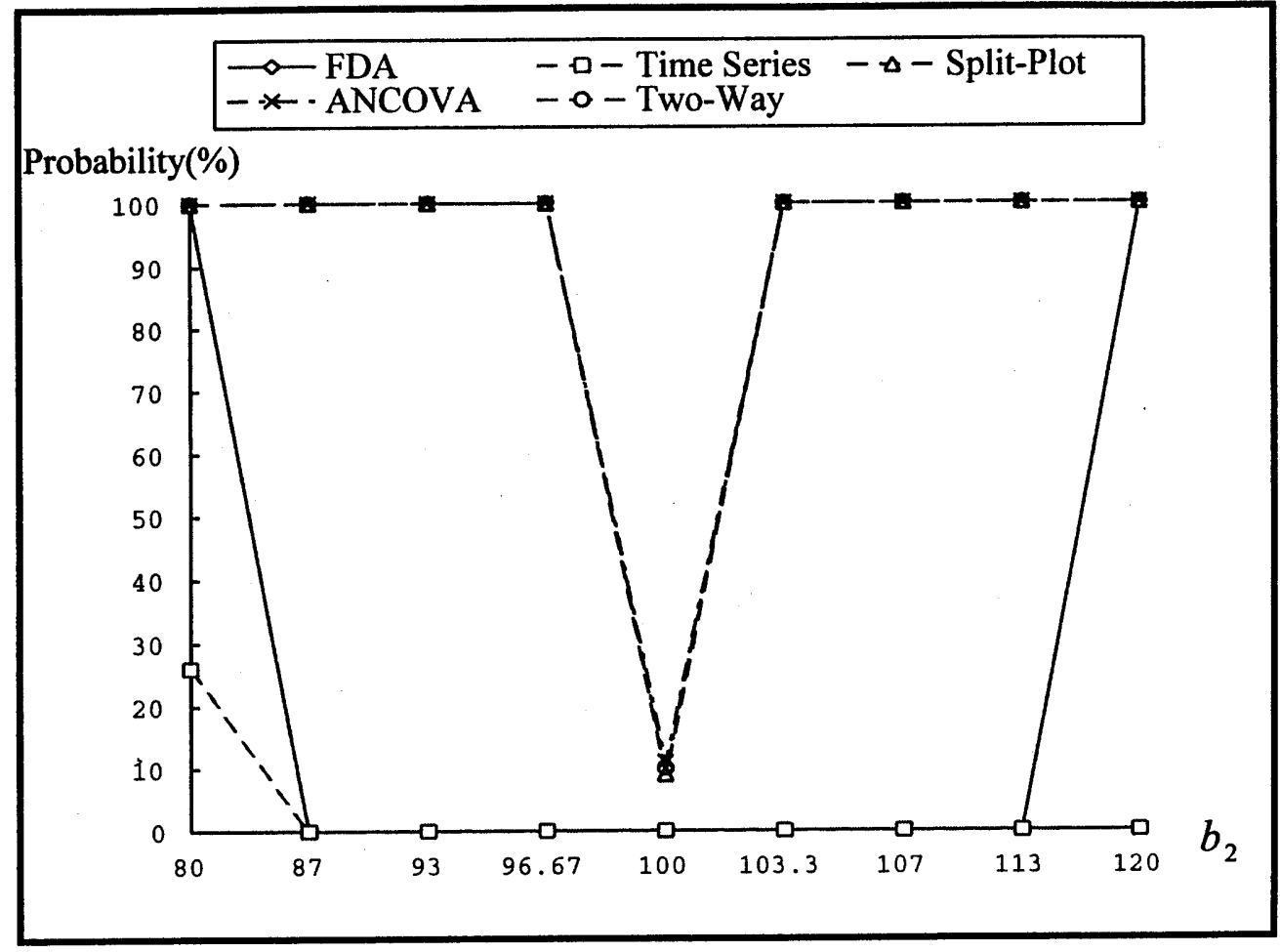

FIGURE 3. The probability of concluding dissimilarity. $a_{1}=a_{2}=0, b_{1}=100, \sigma=1,0.1 \leq$ $t \leq 0.8$.

selected are $0.10,0.25,0.50,0.75,1.00$, and 2.00 (hour). Denote $Q_{t}(R)$ and $Q_{t}(T)$ to be the percentage dissolved for reference and test at time $t$, respectively. Let $b_{11}=130$ and $b_{12}$ $=-40$ so that $Q_{0.75}(R)=75 \%$ and $Q_{2}(R)=100 \%$. Four criteria for simulating the profile of the test are considered.

1. $Q_{0.75}(T)$ varies, $Q_{2}(T)=Q_{2}(R)=100 \%$ - The range of $b_{21}$ and $b_{22}$ are selected so that $Q_{0.75}(T)$ ranges from 50-100\%. Figures 4.1 and 4.2 give an example of the dissolution profiles. The simulation results are given in Tables 3.1 and 3.2. From Table 3.1, it can be observed that FDA detects no difference when $Q_{0.75}(T)$ is within $10 \%$ of the reference. The probability of dissimilarity jumps to 100 when the difference is $15 \%$. The results of Chow show the same pattern with a detectable difference of $20 \%$. Unlike the linear model, ANCOVA has zero probability when the difference is within $5 \%$. This might be due to the fact that the ANCOVA model used in the simulation is based on a linear relationship. Therefore, the results show inconsistency. The sensitivity of these methods are:

\section{Chow $<$ FDA $<$ ANCOVA $<$ Split-Plot, Two-Way ANOVA.}

When $\sigma$ increases (Table 3.2), the change is similar to the linear model where Chow's method is sensible to the variance while the others are not. This pattern is observed throughout the simulation study for the second order model,

2. $Q_{2}(T)$ varies, $Q_{0.75}(T)=Q_{0.75}(R)=75 \%$-Examples of the dissolution profiles are shown in Figures 5.1 and 5.2, where $b_{21}$ and $b_{22}$ are selected so that $Q_{2}(T)$ ranges from 80-105. 


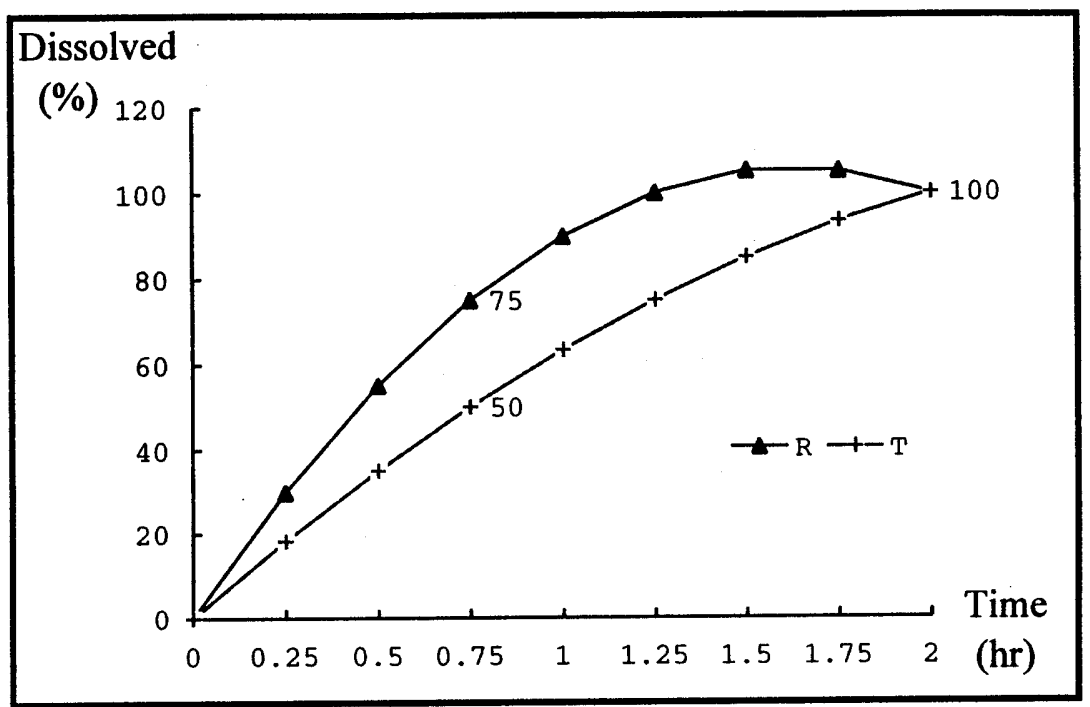

FIGURE 4.1. The dissolution profile of $R\left(Q_{0.75}(R)=75 \%, Q_{2}(R)=100 \%\right)$, and $T\left(Q_{0.75}(T)\right.$ $\left.=50 \%, Q_{2}(T)=100 \%\right)$.

In the study range, FDA and Chow detect no difference (see Table 4.1). ANCOVA cannot observe the difference when $Q_{2}(T)$ between $\mathrm{R}$ and $\mathrm{T}$ is within $10 \%$. Split-plot and twoway ANOVA are consistent with the results of (1), except for a lower probability when two profiles are close (within 1\%). The relationship is:

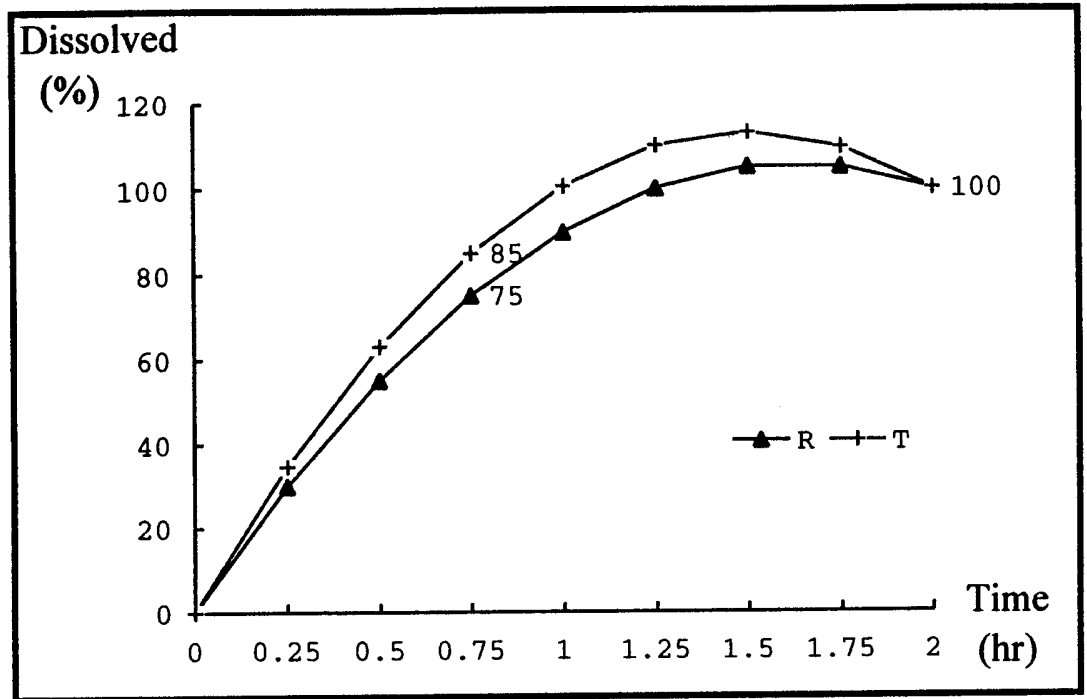

FIGURE 4.2. The dissolution profile of $R\left(Q_{0.75}(R)=75 \%, Q_{2}(R)=100 \%\right)$, and $T\left(Q_{0.75}(T)\right.$ $\left.=85 \%, Q_{2}(T)=100 \%\right)$. 
TABLE 3.1

Probability of Concluding Dissimilarity if $Q_{0.75}(T)$ Varies, $Q_{0.75}(R)=75 \%, Q_{2}(T)=Q_{2}(R)=100 \%, t=0.1,0.25,0.5,0.75,1,2, \sigma=1$

\begin{tabular}{rrrrrrrrrrr}
\hline & \multicolumn{4}{c}{ Test } & \multicolumn{5}{c}{$\begin{array}{c}\text { Time } \\
\text { Numberies }\end{array}$} \\
\cline { 2 - 5 } & $Q_{0.75}$ & $Q_{2}$ & \multicolumn{1}{c}{$b_{21}$} & \multicolumn{1}{c}{$b_{22}$} & FDA & $\begin{array}{c}\text { Split- } \\
\text { Q }=75\end{array}$ & Plot & ANCOVA & $\begin{array}{r}\text { Two- } \\
\text { Way }\end{array}$ \\
\hline 1 & 50 & 100 & 76.7 & -13.3 & 100 & 100 & 100 & 100 & 100 \\
2 & 55 & 100 & 87.3 & -18.7 & 100 & 100 & 100 & 100 & 100 \\
3 & 60 & 100 & 98.0 & -24.0 & 100 & 0 & 100 & 100 & 100 \\
4 & 65 & 100 & 108.7 & -29.3 & 0 & 0 & 100 & 100 & 100 \\
5 & 70 & 100 & 119.3 & -34.7 & 0 & 0 & 100 & 0 & 100 \\
6 & 75 & 100 & 130.0 & -40.0 & 0 & 0 & 10 & 0 & 10 \\
7 & 80 & 100 & 140.7 & -45.3 & 0 & 0 & 100 & 0 & 100 \\
8 & 85 & 100 & 151.3 & -50.7 & 0 & 0 & 100 & 97 & 100 \\
9 & 90 & 100 & 162.0 & -56.0 & 100 & 0 & 100 & 100 & 100 \\
10 & 95 & 100 & 172.7 & -61.3 & 100 & 0 & 100 & 100 & 100 \\
11 & 100 & 100 & 183.3 & -66.7 & 100 & 100 & 100 & 100 & 100 \\
\hline
\end{tabular}

FDA

Chow $<$ ANCOVA $<$ Split-plot, Two-way ANOVA.

When $\sigma$ increases, ANCOVA has a smaller probability than that of Chow as shown in Table 4.2,

3. $b_{22}$ varies, $b_{21}=b_{11}$-Varying $b_{22}$ results in changing both $Q_{0.75}(T)$ and $Q_{2}(T)$. The results are actually a combination of (1) and (2). Still, FDA and Chow's methods are not as sensitive as other methods (see Table 5.1).

When $\sigma$ is small, the relationship is

\section{FDA}

Chow $<$ ANCOVA $<$ Split-plot, Two-way ANOVA.

When $\sigma$ increase, then FDA $<$ Chow as shown in Table 5.2,

TABLE 3.2

Probability of Concluding Dissimilarity if $Q_{0.75}(T)$ Varies, $Q_{0.75}(R)=75 \%, Q_{2}(T)=Q_{2}(R)=100 \%, t=0.1,0.25,0.5,0.75,1,2, \sigma=6$

\begin{tabular}{|c|c|c|c|c|c|c|c|c|c|}
\hline \multirow[b]{2}{*}{ Number } & \multicolumn{4}{|c|}{ Test } & \multirow[b]{2}{*}{ FDA } & \multirow{2}{*}{$\begin{array}{c}\text { Time } \\
\text { Series } \\
\mathrm{Q}=75\end{array}$} & \multirow{2}{*}{$\begin{array}{c}\text { Split- } \\
\text { Plot }\end{array}$} & \multirow[b]{2}{*}{ ANCOVA } & \multirow{2}{*}{$\begin{array}{l}\text { Two- } \\
\text { Way }\end{array}$} \\
\hline & $Q_{0.75}$ & $Q_{2}$ & $b_{21}$ & $b_{22}$ & & & & & \\
\hline 1 & 50 & 100 & 76.7 & -13.3 & 100 & 100 & 100 & 100 & 100 \\
\hline 2 & 55 & 100 & 87.3 & -18.7 & 100 & 97 & 100 & 100 & 100 \\
\hline 3 & 60 & 100 & 98.0 & -24.0 & 85 & 72 & 100 & 100 & 100 \\
\hline 4 & 65 & 100 & 108.7 & -29.3 & 0 & 55 & 100 & 88 & 100 \\
\hline 5 & 70 & 100 & 119.3 & -34.7 & 0 & 47 & 89 & 3 & 91 \\
\hline 6 & 75 & 100 & 130.0 & -40.0 & 0 & 46 & 10 & 0 & 10 \\
\hline 7 & 80 & 100 & 140.7 & -45.3 & 0 & 54 & 87 & 1 & 88 \\
\hline 8 & 85 & 100 & 151.3 & -50.7 & 1 & 70 & 100 & 51 & 100 \\
\hline 9 & 90 & 100 & 162.0 & -56.0 & 82 & 84 & 100 & 99 & 100 \\
\hline 10 & 95 & 100 & 172.7 & -61.3 & 100 & 96 & 100 & 100 & 100 \\
\hline 11 & 100 & 100 & 183.3 & -66.7 & 100 & 100 & 100 & 100 & 100 \\
\hline
\end{tabular}




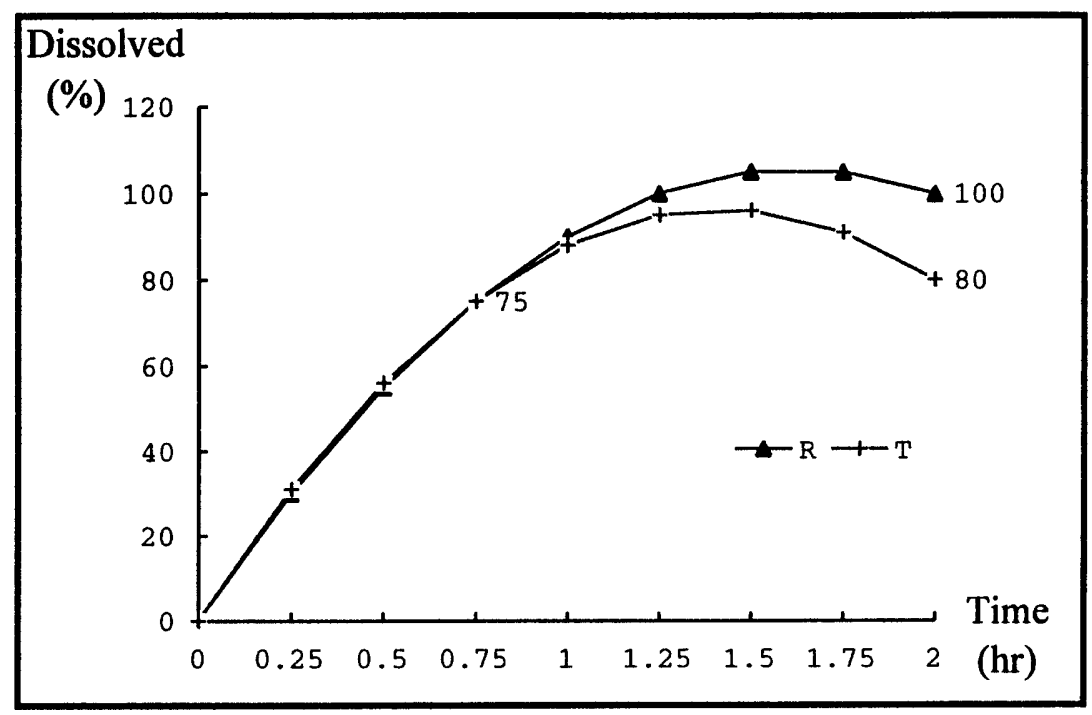

FIGURE 5.1. The dissolution profile of $R\left(Q_{0.75}(R)=75 \%, Q_{2}(R)=100 \%\right)$, and $T\left(Q_{0.75}(T)\right.$ $\left.=75 \%, Q_{2}(T)=80 \%\right)$.

4. $b_{21}$ varies, $b_{22}=b_{12}$ - In this setting, the change of the profile is slower than (3). The results are presented in Tables 6.1 and 6.2, which show a similar pattern to (3).

\section{SUMMARY AND DISCUSSION}

In cases where the dissolution profiles are linear or could be approximated linearly, if the two profiles are in fact identical, FDA's similarity factor is not as sensitive as Chow's is for

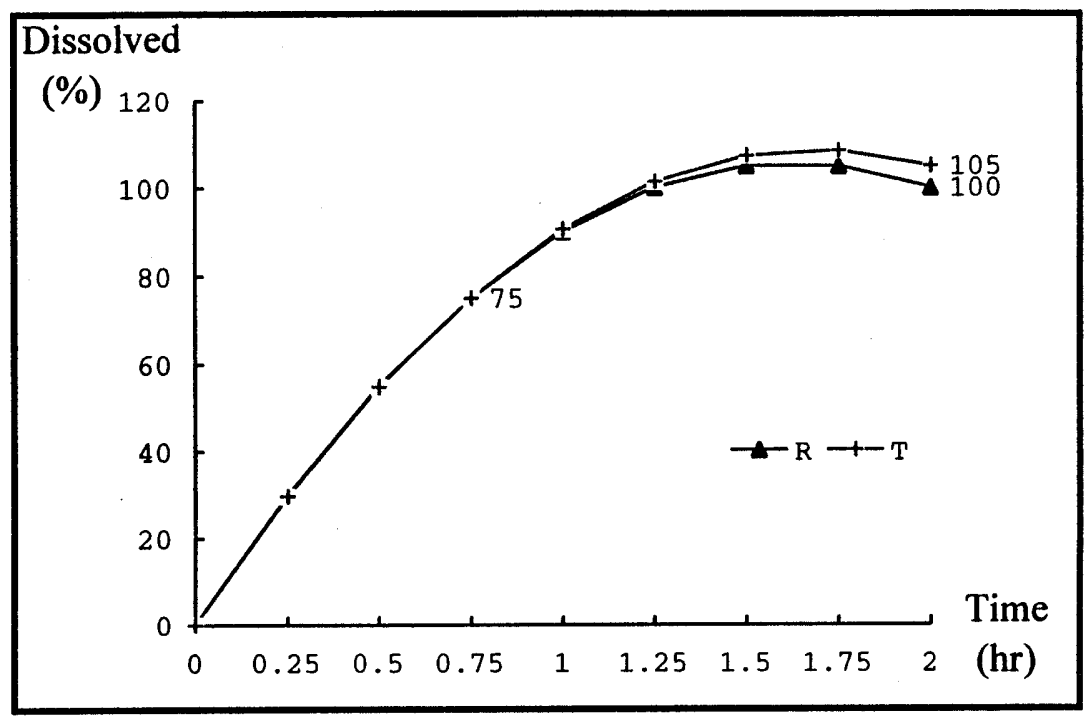

FIGURE 5.2. The dissolution profile of $R\left(Q_{0.75}(R)=75 \%, Q_{2}(R)=100 \%\right.$, and $T\left(Q_{0.75}(T)=\right.$ $\left.75 \%, Q_{2}(T)=105 \%\right)$. 
TABLE 4.1

Probability of Concluding Dissimilarity if $Q_{2}(T)$ Varies, $Q_{2}(R)=100 \%, Q_{0.75}(T)=Q_{0.75}(R)=75 \%, t=0.1,0.25,0.5,0.75,1,2, \sigma=1$

\begin{tabular}{|c|c|c|c|c|c|c|c|c|c|}
\hline \multirow[b]{2}{*}{ Number } & \multicolumn{4}{|c|}{ Test } & \multirow[b]{2}{*}{ FDA } & \multirow{2}{*}{$\begin{array}{l}\text { Time } \\
\text { Series } \\
Q=75\end{array}$} & \multirow{2}{*}{$\begin{array}{l}\text { Split- } \\
\text { Plot }\end{array}$} & \multirow[b]{2}{*}{ ANCOVA } & \multirow{2}{*}{$\begin{array}{l}\text { Two } \\
\text { Way }\end{array}$} \\
\hline & $Q_{0.75}$ & $Q_{2}$ & $b_{21}$ & $b_{22}$ & & & & & \\
\hline 1 & 75 & 80 & 136.0 & -48.0 & 0 & 0 & 100 & 100 & 100 \\
\hline 2 & 75 & 85 & 134.5 & -46.0 & 0 & 0 & 100 & 48 & 100 \\
\hline 3 & 75 & 90 & 133.0 & -44.0 & 0 & 0 & 100 & 0 & 100 \\
\hline 4 & 75 & 95 & 131.5 & -42.0 & 0 & 0 & 100 & 0 & 100 \\
\hline 5 & 75 & 97 & 130.9 & -41.2 & 0 & 0 & 100 & 0 & 100 \\
\hline 6 & 75 & 99 & 130.3 & -40.4 & 0 & 0 & 45 & 0 & 44 \\
\hline 7 & 75 & 100 & 130.0 & -40.0 & 0 & 0 & 10 & 0 & 10 \\
\hline 8 & 75 & 101 & 129.7 & -39.6 & 0 & 0 & 44 & 0 & 46 \\
\hline 9 & 75 & 103 & 129.1 & -38.8 & 0 & 0 & 100 & 0 & 100 \\
\hline 10 & 75 & 105 & 128.5 & -38.0 & 0 & 0 & 100 & 0 & 100 \\
\hline
\end{tabular}

TABLE 4.2

Probability of Concluding Dissimilarity if $Q_{2}(T)$ Varies, $Q_{2}(R)=100 \%, Q_{0.75}(T)=Q_{0.75}(R)=75 \%, \mathrm{t}=0.1,0.25,0.5,0.75,1,2, \sigma=6$

\begin{tabular}{|c|c|c|c|c|c|c|c|c|c|}
\hline \multirow[b]{2}{*}{ Number } & \multicolumn{4}{|c|}{ Test } & \multirow[b]{2}{*}{ FDA } & \multirow{2}{*}{$\begin{array}{l}\text { Time } \\
\text { Series } \\
Q=75\end{array}$} & \multirow{2}{*}{$\begin{array}{l}\text { Split- } \\
\text { Plot }\end{array}$} & \multirow[b]{2}{*}{ ANCOVA } & \multirow{2}{*}{$\begin{array}{l}\text { Two } \\
\text { Way }\end{array}$} \\
\hline & $Q_{0.75}$ & $Q_{2}$ & $b_{21}$ & $b_{22}$ & & & & & \\
\hline 1 & 75 & 80 & 136.0 & -48.0 & 8 & 46 & 100 & 89 & 100 \\
\hline 2 & 75 & 85 & 134.5 & -46.0 & 0 & 46 & 100 & 35 & 100 \\
\hline 3 & 75 & 90 & 133.0 & -44.0 & 0 & 45 & 89 & 3 & 90 \\
\hline 4 & 75 & 95 & 131.5 & -42.0 & 0 & 43 & 35 & 0 & 36 \\
\hline 5 & 75 & 97 & 130.9 & -41.2 & 0 & 44 & 16 & 0 & 16 \\
\hline 6 & 75 & 99 & 130.3 & -40.4 & 0 & 46 & 11 & 0 & 12 \\
\hline 7 & 75 & 100 & 130.0 & -40.0 & 0 & 46 & 10 & 0 & 10 \\
\hline 8 & 75 & 101 & 129.7 & -39.6 & 0 & 46 & 11 & 0 & 10 \\
\hline 9 & 75 & 103 & 129.1 & -38.8 & 0 & 48 & 19 & 0 & 19 \\
\hline 10 & 75 & 105 & 128.5 & -38.0 & 0 & 46 & 36 & 0 & 36 \\
\hline
\end{tabular}

TABLE 5.1

Probability of Concluding Dissimilarity if $\boldsymbol{b}_{22}$ Varies, $b_{21}=b_{11}, Q_{0.75}(R)=75 \%, Q_{2}(R)=100 \%, t=0.1,0.25,0.5,0.75,1,2, \sigma=1$

\begin{tabular}{|c|c|c|c|c|c|c|c|c|}
\hline \multirow[b]{2}{*}{ Number } & \multicolumn{3}{|c|}{ Test } & \multirow[b]{2}{*}{ FDA } & \multirow{2}{*}{$\begin{array}{l}\text { Time } \\
\text { Series } \\
Q=75\end{array}$} & \multirow{2}{*}{$\begin{array}{l}\text { Split- } \\
\text { Plot }\end{array}$} & \multirow[b]{2}{*}{ ANCOVA } & \multirow{2}{*}{$\begin{array}{l}\text { Two } \\
\text { Way }\end{array}$} \\
\hline & $Q_{0.75}$ & $Q_{2}$ & $b_{22}$ & & & & & \\
\hline 1 & 72 & 80 & -45 & 0 & 0 & 100 & 100 & 100 \\
\hline 2 & 73 & 84 & -44 & 0 & 0 & 100 & 100 & 100 \\
\hline 3 & 73 & 88 & -43 & 0 & 0 & 100 & 0 & 100 \\
\hline 4 & 74 & 92 & -42 & 0 & 0 & 100 & 0 & 100 \\
\hline 5 & 74 & 96 & -41 & 0 & 0 & 100 & 0 & 100 \\
\hline 6 & 75 & 100 & -40 & 0 & 0 & 10 & 0 & 10 \\
\hline 7 & 76 & 104 & -39 & 0 & 0 & 100 & 0 & 100 \\
\hline
\end{tabular}


TABLE 5.2

Probability of Concluding Dissimilarity if $\boldsymbol{b}_{22}$ Varies, $b_{21}=b_{11}, Q_{0.75}(R)=75 \%, Q_{2}(R)=100 \%, t=0.1,0.25,0.5,0.75,1,2, \sigma=6$

\begin{tabular}{ccccccccc}
\hline & \multicolumn{3}{c}{ Test } & \multicolumn{5}{c}{$\begin{array}{c}\text { Time } \\
\text { Series }\end{array}$} \\
\cline { 2 - 4 } Number & $Q_{0.75}$ & $Q_{2}$ & $b_{22}$ & FDA & $\begin{array}{c}\text { Split- } \\
\mathbf{Q}=75\end{array}$ & Plot & ANCOVA & $\begin{array}{c}\text { Two- } \\
\text { Way }\end{array}$ \\
\hline 1 & 72 & 80 & -45 & 12 & 47 & 100 & 93 & 100 \\
2 & 73 & 84 & -44 & 1 & 44 & 100 & 57 & 100 \\
3 & 73 & 88 & -43 & 0 & 48 & 99 & 13 & 99 \\
4 & 74 & 92 & -42 & 0 & 48 & 74 & 1 & 74 \\
5 & 74 & 96 & -41 & 0 & 45 & 28 & 0 & 28 \\
6 & 75 & 100 & -40 & 0 & 46 & 10 & 0 & 10 \\
7 & 76 & 104 & -39 & 0 & 47 & 27 & 0 & 29 \\
\hline
\end{tabular}

TABLE 6.1

Probability of Concluding Dissimilarity if $\boldsymbol{b}_{21}$ Varies,

$b_{22}=b_{12}, Q_{0.75}(R)=75 \%, Q_{2}(R)=100 \%, t=0.1,0.25,0.5,0.75,1,2, \sigma=1$

\begin{tabular}{|c|c|c|c|c|c|c|c|c|}
\hline \multirow[b]{2}{*}{ Number } & \multicolumn{3}{|c|}{ Test } & \multirow[b]{2}{*}{ FDA } & \multirow{2}{*}{$\begin{array}{l}\text { Time } \\
\text { Series } \\
Q=75\end{array}$} & \multirow{2}{*}{$\begin{array}{l}\text { Split- } \\
\text { Plot }\end{array}$} & \multirow[b]{2}{*}{ ANCOVA } & \multirow{2}{*}{$\begin{array}{l}\text { Two } \\
\text { Way }\end{array}$} \\
\hline & $Q_{0.75}$ & $Q_{2}$ & $b_{21}$ & & & & & \\
\hline 1 & 71 & 90 & 125 & 0 & 0 & 100 & 0 & 100 \\
\hline 2 & 72 & 92 & 126 & 0 & 0 & 100 & 0 & 100 \\
\hline 3 & 73 & 94 & 127 & 0 & 0 & 100 & 0 & 100 \\
\hline 4 & 74 & 96 & 128 & 0 & 0 & 100 & 0 & 100 \\
\hline 5 & 74 & 98 & 129 & 0 & 0 & 100 & 0 & 100 \\
\hline 6 & 75 & 100 & 130 & 0 & 0 & 10 & 0 & 10 \\
\hline 7 & 76 & 102 & 131 & 0 & 0 & 100 & 0 & 100 \\
\hline 8 & 77 & 104 & 132 & 0 & 0 & 100 & 0 & 100 \\
\hline 9 & 77 & 106 & 133 & 0 & 0 & 100 & 0 & 100 \\
\hline
\end{tabular}

TABLE 6.2

Probability of Concluding Dissimilarity if $b_{21}$ Varies, $b_{22}=b_{12}, Q_{0.75}(R)=75 \%, Q_{2}(R)=100 \%, t=0.1,0.25,0.5,0.75,1,2, \sigma=6$

\begin{tabular}{|c|c|c|c|c|c|c|c|c|}
\hline \multirow[b]{2}{*}{ Number } & \multicolumn{3}{|c|}{ Test } & \multirow[b]{2}{*}{ FDA } & \multirow{2}{*}{$\begin{array}{l}\text { Time } \\
\text { Series } \\
Q=75\end{array}$} & \multirow{2}{*}{$\begin{array}{l}\text { Split- } \\
\text { Plot }\end{array}$} & \multirow[b]{2}{*}{ ANCOVA } & \multirow{2}{*}{$\begin{array}{l}\text { Two- } \\
\text { Way }\end{array}$} \\
\hline & $Q_{0.75}$ & $Q_{2}$ & $b_{21}$ & & & & & \\
\hline 1 & 71 & 90 & 125 & 0 & 47 & 99 & 9 & 99 \\
\hline 2 & 72 & 92 & 126 & 0 & 47 & 90 & 2 & 91 \\
\hline 3 & 73 & 94 & 127 & 0 & 46 & 70 & 0 & 72 \\
\hline 4 & 74 & 96 & 128 & 0 & 49 & 40 & 0 & 41 \\
\hline 5 & 74 & 98 & 129 & 0 & 46 & 17 & 0 & 18 \\
\hline 6 & 75 & 100 & 130 & 0 & 46 & 10 & 0 & 10 \\
\hline 7 & 76 & 102 & 131 & 0 & 49 & 18 & 0 & 19 \\
\hline 8 & 77 & 104 & 132 & 0 & 49 & 40 & 0 & 42 \\
\hline 9 & 77 & 106 & 133 & 0 & 51 & 68 & 0 & 71 \\
\hline
\end{tabular}


variation changes in the amount of dissolution. Other methods have the probability to declare dissimilarity of $10 \%$. If two drugs are different in the initial amount of dissolution but the same in the dissolution rate, then both FDA and Chow have a lower probability of detecting the difference than other methods. In particular, FDA's method cannot detect any difference until the initial amount dissolved between two drug products is $20 \%$ apart. Other methods are very sensitive to the differences. The probability is $100 \%$ in the simulation even for a slight difference (eg, $2.5 \%$ difference), and the probability is a decreasing function of $\sigma$. If two drug products have the same initial dissolution but differ in the dissolution rate, neither the FDA nor the Chow method are as sensitive as others for small variation. In general, FDA's method has a smaller probability than Chow's method regardless of the variation. If the variance is large, then the probability of declaring a difference with Chow's method is even larger than other methods no matter what change exists in the initial amount or rate of dissolution.

In cases where dissolution profiles are quadratic or can be approximated by a quadratic model, if the amount dissolved is the same for two drug products at two boundaries but different in the dissolving rate, neither the FDA method nor the Chow method are sensitive to the differences and have zero probability of declaring a difference of less than 15\% (FDA) or 20\% (Chow). When the variation is large, Chow has a large probability of declaring a difference even when two profiles are identical (eg, probability $=50 \%$ for $\sigma=6$ ) but FDA stays the same. The other methods detect the difference right away (100\% for even a slight difference). If two profiles are the same before 45 minutes and both have $Q_{0.75}=75 \%$ but differ afterwards, the same pattern as the earlier case is observed with FDA and Chow and they are even less sensitive to the difference. If two profiles are different except for the origin in the study range where the difference between $\mathrm{R}$ and $\mathrm{T}$ are within $5 \%$ at $Q_{0.75}$ and $Q_{2}$, FDA and Chow both have zero probability of detecting the difference when the variation is small. The same pattern is observed for Chow for its sensitivity to the variation (eg, 50\% for $\sigma=6$ and $75 \%$ for $\sigma=8$ even when two profiles are identical). For the second order model, ANCOVA has a small probability of declaring the difference, which may due to its linear relationship assumption. The sensitivity of split-plot and two-way ANOVA are decreasing functions of variation.

In general, the FDA and Chow methods are more relaxed than the others. Although FDA's similarity factor is easy to manipulate and results do not change if the role of test and reference interchange, it, however, lacks scientific justification and has the following disadvantages:

1. It is not based on an hypothesis testing procedure, therefore, there is no measurement of the error (Type I or II errors) associated,

2. It is a function of the pair difference between two drug products. If the time points selected for two drug products are different, the method could not be applied, and

3. The measurement is an average of differences observed. After the asymptote is achieved, the differences could be fairly small. In the guideline, no time point selection is specified. If the experimenters select a large number of time points after the asymptote is achieved, then the average difference could be small. In consequence, $f_{2}$ will tend to have a value between 50 and 100 which results in similarity although two profiles could be very different.

Chow's method has an advantage in that it defines the equivalence limits for similarity based on $Q$ value specified in the USP/NF. It also takes into account that the dissolution series observed are in fact dependent and the dependency is a decreasing function of time, as opposed to Gill's method where the relationship is assumed to be constant over time. It, however, has the following disadvantages: 
1. If $Q$ is not specified, the method is difficult to implement in practice,

2. The method is based on relative dissolution that requires the same number of time points and the same number of locations (eg, baskets) for both drug products in the experiment. If either one is different, the method could not be applied,

3. The response is based on the ratio of test over reference. If the role of two drug products is interchanged in the computation, the resulting confidence interval will vary. In consequence, it might lead to a different conclusion, and

4. It is very sensitive to the variation. If the variation is large, the probability of declaring similarity is large even when the underlying distribution of two profiles is identical.

In this research, the authors also extend Chow's method into the auto correlation (2) model, which takes into account the correlation of more than one time point. The results achieved are very similar to the auto correlation (1) model with a slightly wider confidence interval. For example, the interval derived from the auto correlation (2) model for the data in Tsong and Hammerstrom (5) is $(86.71 \%, 112.89 \%)$ which is within two tenth points of the auto correlation (1) model as shown in the "Methods" section.

For Gill's method, the advantage is that it reflects the fact that correlation between the amount dissolved for the same product exists. This assumption, however, seems oversimplified since it is assumed to be constant. In addition, due to the algorithm used for the hypothesis testing, the Type I error is twice as high as it should be since dissimilarity could be concluded in two cases, that is, if the treatment by time interaction exists or if the difference between treatments is found in the reduced model. Moreover, using time as a class variable in the analysis dramatically reduces the degree of freedom and this is also a disadvantage of the method.

It has been observed that ANCOVA and two-way ANOVA are often used in comparing dissolution profiles. ANCOVA especially is preferred by most of the practitioners since it can estimate the dissolution rate of drug products other than testing the similarity of the profiles. It should be noted that the method is not appropriate if the dissolution profiles are not linear or if only a few time points are tested. Moreover, the correlation nature of the dissolution series within the same product violates the underlying independent assumptions required for applying either of the methods. The validity of the results is, therefore, questionable.

This paper investigates the sensibility of commonly used methods for analyzing and evaluating the similarity of the dissolution profiles of two drug products. Note that a good analysis actually results from a good design. A valid experimental design is indeed essential in dissolution testing. Before the experiment is conducted, one should carefully plan the experiment such as the suitability of the apparatus (eg, rotating basket for capsules, paddle for tablets), the choice of media, response measured, location of dissolution basket $(2 \times(3,3)$ or $(6,0)(0,6)$ for $(\mathrm{R}, \mathrm{T})$, and so forth. To date, several issues exist in the design of dissolution testing that need further investigation. These issues include the selection of the number of time points, sampling time, number of replicates, the choice of media, and so forth. Moreover, how to scientifically assess the in vivo and in vitro correlation $(15,16)$ remains to be solved.

Acknowledgments-The authors thank Dr. J. P. Liu for his suggestions and comments on this work.

\section{REFERENCES}

1. FDA. Guidance for industry: immediate release solid oral dosage forms scale-up and postapproval changes: chemistry, manufacturing and controls, in vitro dissolution testing, and in vivo bioequivalence documentation. Rockville, MD: Food and Drug Administration; November 15, 1995. 
2. Chow SC. Statistical comparison between dissolution profiles of drug products. Presented at Department of Health, Executive Yuan, Taipei, Taiwan, 1995.

3. Chow SC, Liu JP. Statistical Design and Analysis in Pharmaceutical Science. New York: Marcel Dekker; 1995.

4. USP XIII.

5. Tsong Y, Hammerstrom T. Statistical issues in drug quality control based on dissolution testing. Proceedings of Biopharmaceuticl section of American Statistical Association, 1994;295-300.

6. Dawoodbhai S, et. al. Optimization of tablet formulation containing talc. Drug Dev Ind Pharm. 1991;17: 1343-1371.

7. Pena Romero A, et. al. Water uptake and force development in an optimized prolonged release formulation. Int J Pharm. 1991;73:239-248.

8. Langenbucher F. Linearization of dissolution rate curves by the Weilbull distribution. J Pharm Pharmacol. 1972;24:979-981.

9. Kervinen L, Yliruusi J. Modelling S-Shaped dissolution curves. Int J Pharm. 1993;92:115-122.

10. Montgomery D. Design and Analysis of Experiments. 3rd ed. New York: John Wiley and Sons; 1991.

11. Mauger JW, et al. On the analysis of dissolution data. Drug Dev Ind Pharm. 1986:12(7):969-992.

12. Gill JL. Repeated measurement: split-plot trend analysis versus analysis of first differences. Biometrics. 1988; 44:289-297.

13. Tsong Y. Statistical assessment of mean differences between two dissolution data sets. Presented at the DIA Dissolution Workshop, Rockville, Maryland, 1995.

14. Box GEP, et al. Time Series Analysis, Forecasting and Control. 3rd ed. Englewood Cliffs, NJ: Prentice Hall; 1994.

15. Shah VP, Lesko LJ. Current challenges and future regulatory directions in in vitro dissolution. Drug Info J. 1995;885-891.

16. Leeson LJ. In vitro/in vivo correlations. Drug Info J. 1995;903-915. 